\title{
Foil cycling technique for the VESUVIO spectrometer operating in the resonance detector configuration
}

\author{
E. M. Schooneveld, J. Mayers, and N. J. Rhodes \\ ISIS Facility, Rutherford Appleton Laboratory, Chilton, Didcot, Oxfordshire OX11 OQX, United Kingdom
}

\begin{abstract}
A. Pietropaolo
Consiglio Nazionale delle Ricerche-Istituto Nazionale per la Fisica della Materia, Università degli Studi di Roma Tor Vergata, Via della Ricerca Scientifica 1, 00133 Roma, Italy

C. Andreani and R. Senesi

Dipartimento di Fisica, Università degli Studi di Roma "Tor Vergata," Via della Ricerca Scientifica 1, 00133 Roma, Italy

G. Gorini, E. Perelli-Cippo, and M. Tardocchi

Dipartimento di Fisica "G. Occhialini," Università degli Studi di Milano Bicocca, Piazza della Scienza 2, I-20126 Milano, Italy and CNR-INFM, Università degli Studi di Milano Bicocca, Piazza della Scienza 2, I-20126 Milano, Italy
\end{abstract}

(Received 31 March 2006; accepted 8 August 2006; published online 25 September 2006)

This article reports a novel experimental technique, namely, the foil cycling technique, developed on the VESUVIO spectrometer (ISIS spallation source) operating in the resonance detector configuration. It is shown that with a proper use of two foils of the same neutron absorbing material it is possible, in a double energy analysis process, to narrow the width of the instrumental resolution of a spectrometer operating in the resonance detector configuration and to achieve an effective subtraction of the neutron and gamma backgrounds. Preliminary experimental results, obtained from deep inelastic neutron scattering measurements on lead, zirconium hydride, and deuterium chloride samples, are presented. () 2006 American Institute of Physics. [DOI: 10.1063/1.2349598]

\section{INTRODUCTION}

Since the middle 1980s, epithermal neutron scattering experiments are performed at spallation neutron sources where intense fluxes of neutrons in the energy region above $1 \mathrm{eV}$ are available. Inelastic neutron scattering measurements at $\mathrm{eV}$ energies are currently performed at the ISIS spallation source on the VESUVIO inverse geometry spectrometer.

Neutron scattering experiments on VESUVIO are currently performed in two different regimes: (a) at large energy $(\hbar \omega)$ and wave vector $(q)$ transfers [deep inelastic neutron scattering (DINS)] (Ref. 2) and (b) at large $\hbar \omega$ and low $q$ [high energy inelastic neutron scattering (HINS)]. ${ }^{3}$ DINS is the most relevant experimental technique to study the single particle short time dynamics in condensed matter systems, ${ }^{4,5}$ while HINS allows the investigation of high energy excitations, such as dispersion relations of high energy excitations in magnetic materials, semiconductors, high lying molecular rotational-vibrational states, molecular electronic excitations, and electronic levels in solids.

At present, the VESUVIO spectrometer can operate in both the resonance filter (RF) (Refs. 6 and 7) and the resonance detector (RD) (Refs. 8 and 9) configurations. Both configurations utilize a so-called analyzer foil of a specific material, such as uranium $\left({ }^{238} \mathrm{U}\right)$ and gold $\left({ }^{197} \mathrm{Au}\right)$, in the secondary flight path. This analyzer foil strongly absorbs neutrons within narrow energy intervals around nuclear resonances, thus selecting the energy of the scattered neutrons. In the RF configuration the analyzer foil is cycled in and out of the scattered neutron beam and two measurements are taken, one with the foil between sample and detector and the other with the foil removed. The transmitted beam is recorded by ${ }^{6} \mathrm{Li}$-glass neutron counters and the scattering time of flight spectrum is reconstructed through the filter difference (FD) (Refs. 6 and 7) and/or the double difference (DD) (Refs. 10 and 11) methods. In the RD configuration ${ }^{12,13}$ the analyzer foil is stationary and in close contact with a photon detector. The photon detector tags the arrival time of the absorbed neutron by registering the prompt radiative capture $g$ rays. In this way the instrument responds mainly to the analyzer's resonances, with an efficiency which is mostly independent of neutron energy.

Several studies have been performed in the last six years on VESUVIO, optimizing different combinations of analyzers and photon detectors ${ }^{11,14-19}$ in the RD configuration. These studies have demonstrated that cerium activated yttrium-aluminum-perovskite (YAP) scintillators are the most promising detectors in terms of efficiency and signal to background ratio enhancement. Recently these scintillators have been successfully used for both DINS (Refs. 18 and 19) and HINS (Refs. 20-25) measurements at the new very low angle detector (VLAD) bank installed on VESUVIO in December $2005,{ }^{26}$ demonstrating the effectiveness of the RD for epithermal neutron spectroscopy in the two scattering regimes.

This article introduces a novel experimental technique, namely, the foil cycling technique (FCT). It relies upon the 


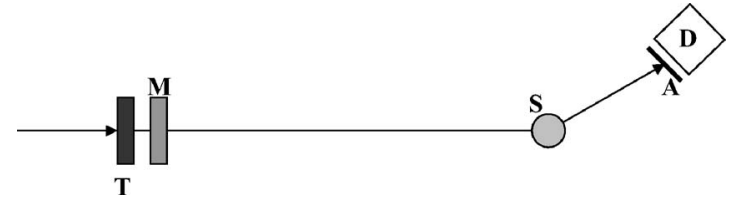

FIG. 1. Schematic layout of VESUVIO operated in the resonance detector (RD) configuration. In this figure $\mathrm{T}$ represents the heavy metal target for the spallation reaction, $\mathrm{M}$ is the moderator, $\mathrm{S}$ is the sample, A represents the energy analyzer, and $\mathrm{D}$ is the photon detector.

use of two foils of the same absorbing material, one stationary analyzer foil and one so-called cycling foil that alternates in and out of the scattered neutron beam, combining the RD and the FD techniques. It is shown that the double energy analysis performed in the FCT provides a substantial narrowing of the spectrometer resolution and an effective neutron/ gamma background subtraction, without a significant loss in counting efficiency.

In Sec. II VESUVIO operating in the RD configuration is briefly described and in Sec. III the FCT is discussed. Sections IV and V report the experimental DINS data from $\mathrm{Pb}, \mathrm{DCl}$, and $\mathrm{ZrH}_{2}$ samples and the summary of the results, respectively. The discussion is presented in Sec. VI.

\section{VESUVIO IN THE RESONANCE DETECTOR CONFIGURATION}

The experiments presented in this work have been performed on VESUVIO operating in the RD configuration, as shown schematically in Fig. 1. The incident neutron beam from a water moderator at $295 \mathrm{~K}$ is characterized by a white energy spectrum, resulting in a peak at about $0.03 \mathrm{eV}$ and an $E_{0}^{-0.9}$ tail in the epithermal region, $E_{0}$ being the incident neutron energy. The RD detection system for the measurements consisted of a device which combines an YAP scintillator and a ${ }^{197} \mathrm{Au}$ analyzer foil. YAP is a fast, mechanically rugged, and chemically resistant scintillator material. It is nonhygroscopic, with a density of $5.55 \mathrm{~g} / \mathrm{cm}^{3}$, an effective atomic number $Z=36$, and a light yield of 18000 photons $/ \mathrm{MeV}$. It has a short decay time $\tau=27 \mathrm{~ns}$ and a wavelength of maximum emission of $350 \mathrm{~nm}$. The material is relatively stable over a wide temperature range and the chemical composition is such that no neutron resonances are present in the $1-200 \mathrm{eV}$ energy range of interest.

When the RD configuration is employed at a pulsed neutron source, the measurement of the time of arrival of the $\gamma$ 's, which coincides with the instant of neutron absorption in the foil, allows the determination of the initial neutron energy through the time of flight (TOF) technique.

The acquired signal is a TOF spectrum where the count rate per time bin is given, with good degree of accuracy, by the approximated expression ${ }^{11,27}$

$$
C(t)=\frac{\phi\left(E_{0}\right) A_{d} E_{0}}{L_{0}^{3} L_{1}^{2} 4 \pi} 2 \sqrt{\frac{2 E_{0}}{m}} \delta t N_{s} \frac{d^{2} \sigma\left(E_{0}, E_{r}, \vartheta\right)}{d \Omega d E_{r}} \Delta E_{r} \eta_{d}
$$

where $\phi\left(E_{0}\right)$ is the spectrum of the incident neutrons at the energy $E_{0}, A_{d}$ is the analyzer's foil area which determines the geometrical acceptance of the detector, $L_{0}$ and $L_{1}$ are the primary and the scattering flight paths, respectively, while $m$

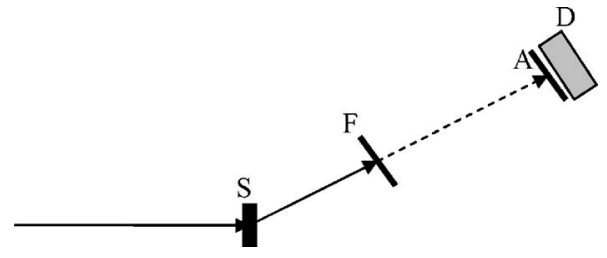

FIG. 2. Schematic of the foil cycling technique (FCT). As in the standard $\mathrm{RD}$ configuration, $\mathrm{S}$ represents the sample, $\mathrm{A}$ the analyzer, and $\mathrm{D}$ the photon detector. The cycling foil, introduced by the FCT, is shown as F.

is the neutron mass. $N_{S}$ is the number of scattering centers in the sample and $d^{2} \sigma\left(E_{0}, E_{r}, \vartheta\right) / d E_{r} d \Omega$ is the sample's double-differential scattering cross section. The quantity $\Delta E_{r}$ is the full width at half maximum (FWHM) of the transfer function of the analyzer ( $E_{r}$ being the resonance energy), while $\eta_{d}$ represents the total detection efficiency.

The neutron resonance absorption cross section for an isolated nucleus at rest is a Lorentzian function of the neutron energy. ${ }^{28}$ However, the absorbing nucleus is embedded in a solid where the effect of crystal lattice motion broadens the resonance absorption profile from a pure Lorentzian line shape. ${ }^{29,30}$ The overall resonance line shape, in the thin foil approximation, ${ }^{9}$ can be described by a Voigt function provided by an integral convolution of the Lorentzian (intrinsic nuclear component) and Gaussian (Doppler component) energy distributions. This analyzer component of the resolution function (referred to as the energy component) has to be convoluted with the other (geometrical and time) components of the spectrometer resolution (which can be well approximated by a Gaussian), thus resulting in a Voigt line shape for the overall resolution function. ${ }^{11,31,32}$

\section{FOIL CYCLING TECHNIQUE}

The width of the resolution function can be narrowed by employing a technique, henceforth called the FCT, described schematically in Fig. 2. It envisages the use of two foils of the same material: the first foil (analyzer) is placed in front of the photon detector and behaves as a standard energy analyzer for the RD configuration (see Fig. 1), while the second foil (cycling foil) is placed between the sample and the analyzer at a given distance from the latter. In the scattering experiment the cycling foil alternates in and out of the scattered neutron beam, leaving the analyzer in front of the photon detector. Subtracting the spectrum acquired with the cycling foil in the foil-in position from the spectrum acquired in the foil-out position produces the final data. The improvement of the resolution function is due to the narrowing of the FWHM of the transfer function using this technique. The FCT may be called a "redundant resolution" technique, one resolving method (the RD) piled upon another (the FD).

The cycling foil acts as a filter and removes neutrons from the scattered beam having energies in the range of $\pm \Delta E_{r}$ near the resonance energy $E_{r}$. When the cycling foil is between the sample and the analyzer, the photon detector registers the $\gamma$ 's produced by neutrons that pass the filter and are absorbed by the analyzer. Photons produced by the cycling foil are much less effectively detected due to the smaller solid angle subtended by the detector. The contribu- 


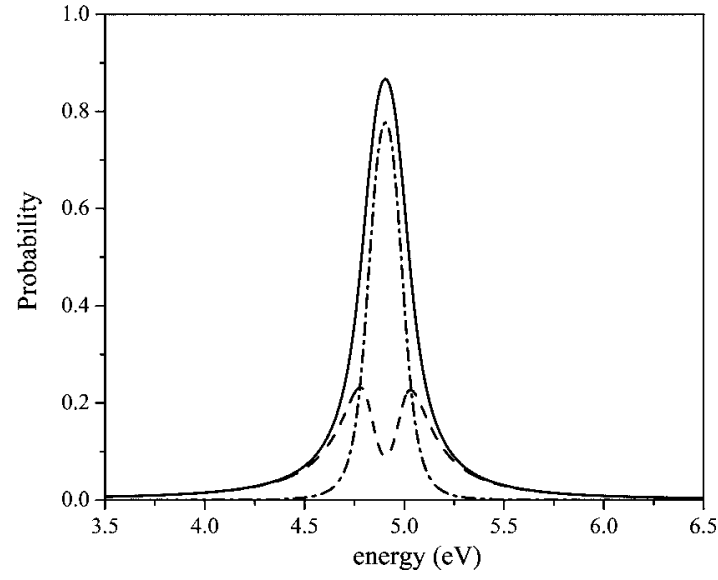

FIG. 3. Transfer functions calculated using $12.5 \mu \mathrm{m}$ thick ${ }^{197} \mathrm{Au}$ foils. Shown are the foil-out (standard RD) transfer function $T_{\text {out }}(E)$ as a continuous line, the foil-in transfer function $T_{\mathrm{in}}(E)$ as a dashed line, and the FCT effective transfer function $T(E)$ as a dashed-dotted line.

tion of $\gamma$ 's produced by the cycling foil is further reduced by the subtraction procedure, as the number of $\gamma$ 's from the cycling foil registered by the detector can be made comparable for the foil-in and foil-out positions.

The improvement of the resolution function can be explained as follows: Consider cycling and analyzer foils of identical thickness $d$. For the foil-out spectrum the transfer function is given by

$$
T_{\text {out }}(E)=1-\exp \left[-N \sigma_{\gamma}(E) d\right],
$$

where $\sigma_{\gamma}(E)$ is the radiative capture cross section as a function of the neutron energy and $N$ the number of absorbing nuclei per unit volume.

The transmission through the cycling foil is given by

$$
T_{\text {filter }}(E)=\exp \left[-N \sigma_{T}(E) d\right],
$$

where the total cross section $\sigma_{T}(E)$ is used to account for all the physical processes which cause removal of neutrons from the scattered beam.

For the foil-in case the complete transfer function is therefore given by the expression

$$
T_{\text {in }}(E)=\exp \left[-N \sigma_{T}(E) d\right]\left\{1-\exp \left[-N \sigma_{\gamma}(E) d\right]\right\} .
$$

Performing the difference between the foil-out and the foil-in transfer functions, an effective transfer function $T(E)$ is yielded, which has the following form:

$$
T(E)=\left\{1-\exp \left[-N \sigma_{\gamma}(E) d\right]\right\}\left\{1-\exp \left[-N \sigma_{T}(E) d\right]\right\} .
$$

Taking into account that, at a resonance, the total cross section $\sigma_{T}(E)$ and the radiative cross section $\sigma_{\gamma}(E)$ are almost identical, the effective transfer function $T(E)$ can be approximated by

$$
T(E) \simeq\left\{1-\exp \left[-N \sigma_{\gamma}(E) d\right]\right\}^{2} .
$$

Hence the effective transfer function, using the FCT, is approximately the square of the transfer function of the RD configuration quoted in Eq. (2). If $1-\exp \left[-N \sigma_{\gamma}(E) d\right]$ were of Gaussian form, then $T(E)$ in Eq. (6) would have a width smaller by a factor of 0.71 (i.e., 29\%) as compared to $T_{\text {out }}(E)$. The change observed in the theoretical calculation of the transfer functions, shown in Fig. 3, is a factor of 0.74, that is, $26 \%$.

Figure 3 shows $T_{\text {out }}(E)$ (continuous line), $T_{\text {in }}(E)$ (dashed line), and $T(E)$ (dashed-dotted line) in the case of $12.5 \mu \mathrm{m}$ thick ${ }^{197} \mathrm{Au}$ analyzer/cycling foils. This thickness is the one employed for the DINS measurements described in the following results.

The transfer function $T(E)$ has a FWHM which is about $26 \%$ narrower than $T_{\text {out }}(E)$, the transfer function of the standard RD. The difference in the peak value of the interaction probability is about $10 \%$. Figure 3 also shows that the FCT transfer function has substantially less pronounced tails than the standard RD transfer function $T_{\text {out }}(E)$, greatly improving the sensitivity for low concentrations of elements of similar masses.

An additional advantage of the FCT is that it provides an inherent background subtraction. Outside the resonance, the total cross section $\sigma_{T}(E)$ is small so that the transmission through the cycling foil $T_{\text {filter }}(E)$ is approximately 1 . Consequently, $T_{\text {out }}(E) \cong T_{\text {in }}(E)$ and $T(E)$, obtained by the subtraction $T_{\text {out }}(E)-T_{\text {in }}(E)$, is very close to zero, enabling a very good background subtraction.

\section{RESULTS}

This section reports the experimental results obtained during a series of DINS experiments performed at the VESUVIO spectrometer on $\mathrm{Pb}, \mathrm{DCl}$, and $\mathrm{ZrH}_{2}$ samples.

\section{A. Lead sample}

DINS measurements on a $\mathrm{Pb}$ reference sample, $2 \mathrm{~mm}$ thick and $100 \mathrm{~cm}^{2}$ in area, have been performed using $12.5 \mu \mathrm{m}$ thick ${ }^{197} \mathrm{Au}$ analyzer/cycling foils and an YAP scintillator detector operating with a lower level discrimination (LLD) threshold of circa $600 \mathrm{keV}$. The photon detector was at a distance $L_{1} \simeq 0.7 \mathrm{~m}$ from the $\mathrm{Pb}$ sample and at a scattering angle $q \vartheta=45^{\circ}$. The analyzer foil was attached to the surface of the detector, while the distance between the cycling foil and analyzer foil was about $0.35 \mathrm{~m}$.

The DINS measurements from $\mathrm{Pb}$ represent a very stringent test to demonstrate the effective narrowing of the transfer function applying the FCT. In fact, for large masses the spectrometer's response is very sensitive to the overall resolution function, such that changes in the transfer function result in appreciable differences between spectra acquired with the standard RD energy analysis and those obtained with the FCT.

Figure 4 shows two DINS spectra in the time region of 300-450 $\mu \mathrm{s}$ : the open dot spectrum represents the recoil peak of the ${ }^{207} \mathrm{~Pb}$ measured at a final neutron energy of $4.906 \mathrm{eV}$. This spectrum was acquired using only the analyzer foil (foil-out measurement). The peak is located at about $380 \mu \mathrm{s}$ and the FWHM of the distribution is about $14 \mu \mathrm{s}$. The background was corrected by subtracting a TOF spectrum acquired by the YAP detector without the cycling and analyzer foils in the scattered beam.

The full dot spectrum represents the foil-in measurement. In this case the same background correction method has been applied as for the foil-out measurement. It shows a dip in the TOF region corresponding to the peak of the recoil 


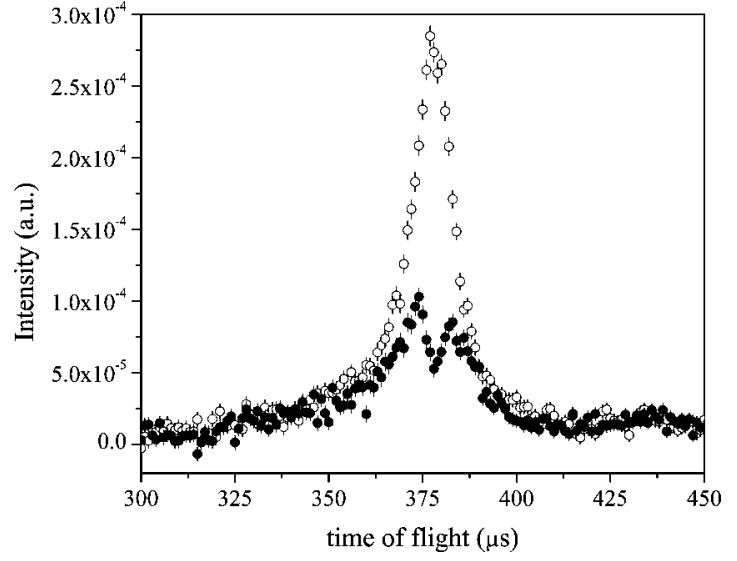

FIG. 4. Experimental TOF spectra from a ${ }^{207} \mathrm{~Pb}$ sample for the foil-out measurement (open dots) and for the foil-in measurement (full dots). These data have been corrected for background using a separate measurement without analyzer and cycling foils.

distribution. This is due to the high attenuation of the $4.906 \mathrm{eV}$ neutrons due to the presence of the cycling foil.

The difference between the two spectra shown in Fig. 4 provides a new recoil spectrum. Figure 5 shows the standard DINS spectrum acquired with the analyzer only (open dots) and the one obtained from the difference of the foil-in and foil-out spectra (full dots). For the full dot spectrum the background subtraction is automatically provided by the difference of the foil-in and foil-out spectra. The difference spectrum has a FWHM of about $8 \mu \mathrm{s}$. The narrower width of the distribution is due to the narrower effective transfer function $T(E)$ [see Eq. (5)] shown in Fig. 3. The background subtraction achieved with the FCT is more effective as can be seen by the absence in Fig. 5 of a very small offset present in Fig. 4. Furthermore the FCT provides a more symmetric time distribution, due to an almost complete cancellation of the $1 / v$ tail, characteristic of the ${ }^{197} \mathrm{Au}$ neutron absorption cross section. The peak intensity $I$ of the difference spectrum is about $25 \%$ lower than the intensity of the standard RD peak (foil out), but the statistical uncertainty in the intensity $\Delta I / I$ is still quite good. In fact, at the recoil peak maximum $\Delta I / I$ is about $3 \%$ for the standard measurement and about

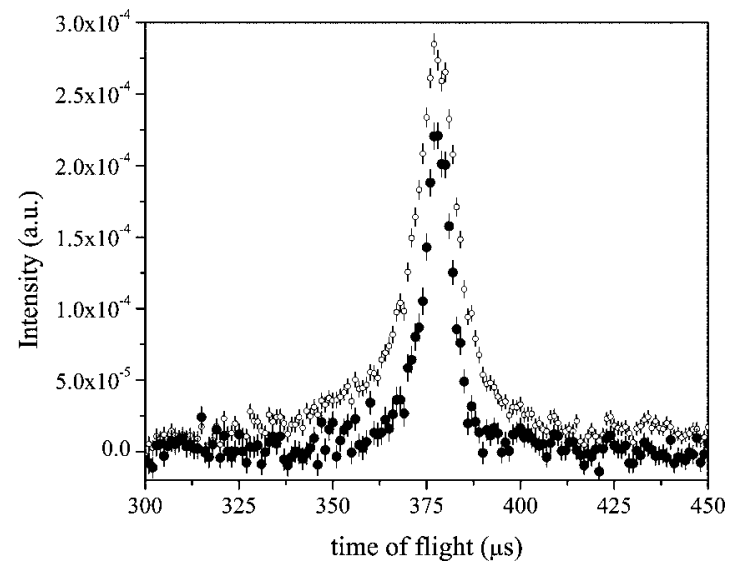

FIG. 5. Experimental TOF spectra from DINS measurements on a ${ }^{207} \mathrm{~Pb}$ sample for the standard RD configuration (open dots) and the FCT (full dots). The improved resolution (narrower peak) obtained with the FCT is clearly visible.

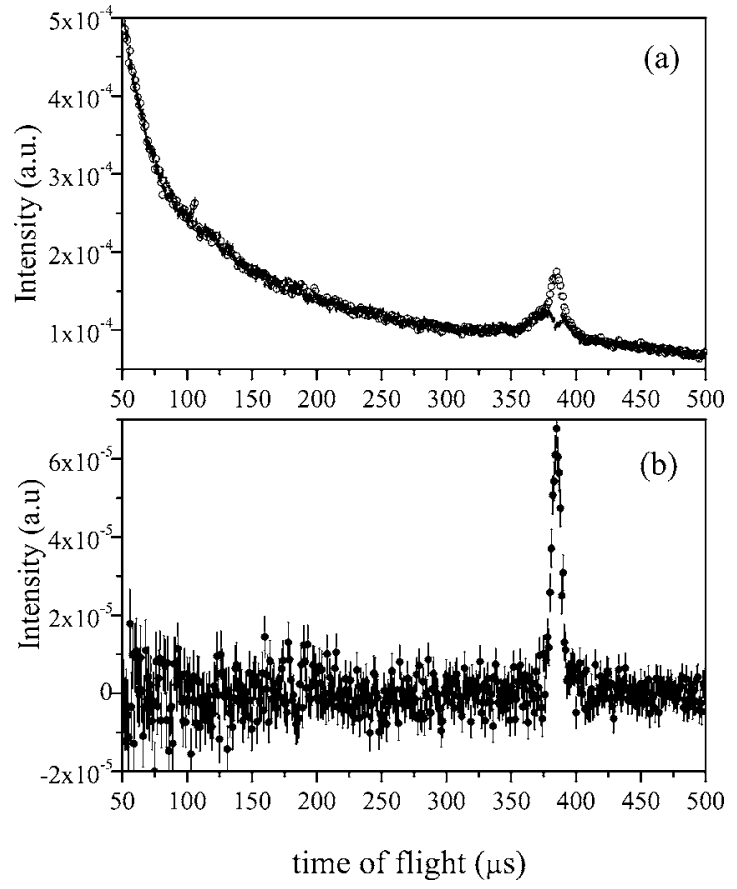

FIG. 6. TOF spectra from a ${ }^{207} \mathrm{~Pb}$ sample in an extended TOF region $(50-500 \mu \mathrm{s})$. (a) Raw data of the foil-out measurement (open dots) and foil-in measurement (full dots), demonstrating the background present in the raw data. (b) Difference between the spectra shown in (a), thus obtained using the FCT. The effectiveness of the background subtraction achieved with the FCT in the whole time of flight region can be appreciated.

$4 \%$ for the FCT spectra. These measurements confirm that the background subtraction obtained by the FCT is very effective in the entire TOF region of interest for DINS experiments. Figure 6(a) shows the foil-in and foil-out DINS spectra obtained with the $\mathrm{Pb}$ sample, in the TOF region of 50-500 $\mu$ s without applying any background correction. Figure 6(b) shows the difference spectrum in the same TOF region, where the quality of the inherent background correction of the FCT can be appreciated.

\section{B. Deuterium chloride sample}

In this experiment the $\mathrm{DCl}$ sample was at a temperature of $T=4.5 \mathrm{~K}$, in thermodynamical equilibrium with its vapor, and enclosed in an aluminum sample container. The measurements have been performed parasitically during a DINS experiment carried out in the RF configuration.

For this experiment an YAP detector was used at a scattering angle $2 \vartheta \simeq 40^{\circ}$ and at $L_{1} \simeq 80 \mathrm{~cm}$. The LLD setting was about $600 \mathrm{keV}$. As in the $\mathrm{Pb}$ measurements, both the analyzer and the cycling foil were metallic foils of ${ }^{197} \mathrm{Au}$ with a thickness $d \simeq 12.5 \mu \mathrm{m}$.

Figure 7(a) shows the DINS spectrum from the DCl sample using only the analyzer foil, i.e., the standard RD configuration. A background correction has been performed by fitting the regions outside the recoil peaks with an appropriate function. ${ }^{19}$ The continuous line is a fit provided by a multipeak fitting procedure. The dashed-dotted line, peaking at about $376 \mu \mathrm{s}$, is the contribution of the ${ }^{35} \mathrm{Cl}+{ }^{27} \mathrm{Al}$ recoil, corresponding to a final neutron energy of $4.906 \mathrm{eV}$ (first ${ }^{197} \mathrm{Au}$ resonance). The dashed line, showing a peak at about $347 \mu \mathrm{s}$, is the recoil peak of ${ }^{2} \mathrm{D}$, corresponding to the same 

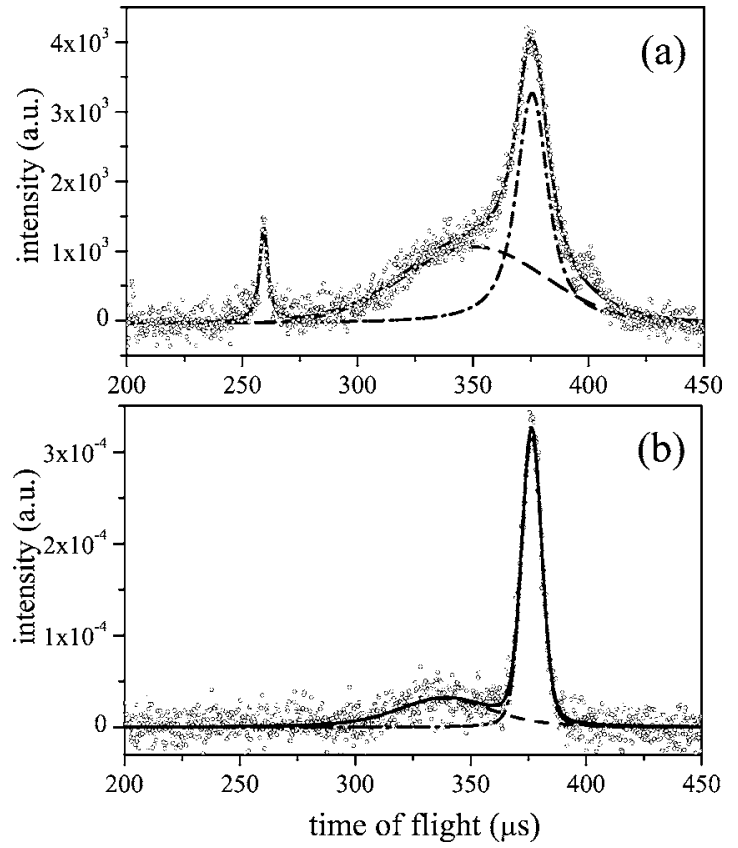

FIG. 7. Results from DINS experiments on a DCl sample in the time region of 200-450 $\mu$ s for (a) the standard RD configuration and (b) the FCT. The continuous line is a multipeak fitting which encompasses the contributions from ${ }^{2} \mathrm{D}$ (dashed line) and ${ }^{35} \mathrm{Cl}+{ }^{27} \mathrm{Al}$ (dashed-dotted line).

final neutron energy. Due the to low mass of the deuterium nucleus and the small scattering angle, the recoil peaks of ${ }^{2} \mathrm{D}$ and ${ }^{35} \mathrm{Cl}+{ }^{27} \mathrm{Al}$ are partially superimposed. The peak located at $260 \mu \mathrm{s}$ is a spurious peak caused by sample environment equipment. The width of the ${ }^{35} \mathrm{Cl}+{ }^{27} \mathrm{Al}$ recoil peak is about $18 \mu$ s FWHM, while the ${ }^{2} \mathrm{D}$ recoil peak has a FWHM of about $52 \mu \mathrm{s}$. Figure 7(b) shows the DINS spectrum of the $\mathrm{DCl}$ sample registered by the YAP detector employing the FCT, thus subtracting the foil-in spectrum. As explained in Sec. III, no separate background correction was required. The spurious peak at $260 \mu$ s, present in Fig. 7(a), is absent in this spectrum. Since this peak does not originate from neutrons with a final energy of $4.906 \mathrm{eV}$, the foil-in subtraction has correctly removed this spurious signal. Other background contributions have also been successfully removed.

In this case the width of the ${ }^{35} \mathrm{Cl}+{ }^{27} \mathrm{Al}$ recoil peak is about $10 \mu$ sWHM, while the FWHM of the ${ }^{2}$ D recoil peak is about $47 \mu \mathrm{s}$. The lower relative peak height of the ${ }^{2} \mathrm{D}$ recoil peak is probably due to a lower $\mathrm{DCl}$ concentration during the FCT measurements, as the data were taken during the filling procedure of the sample container.

The improvement in width, obtained by the FCT, is about $44 \%$ for the ${ }^{35} \mathrm{Cl}+{ }^{27} \mathrm{Al}$ peak and about $10 \%$ for the ${ }^{2} \mathrm{D}$ peak. This is due to the fact that the width of the ${ }^{35} \mathrm{Cl}$ $+{ }^{27} \mathrm{Al}$ recoil peak is mostly dominated by the instrumental resolution while, due to the lower mass of the deuteron, the width of the ${ }^{2} \mathrm{D}$ recoil peak is largely due to the atomic momentum distribution. Furthermore the overlap of the two peaks in the TOF spectrum of Fig. 7(b), which would be less if the scattering angle were higher, is appreciably less as compared to that in Fig. 7(a).

\section{Zirconium hydride sample}

Measurements on a $\mathrm{ZrH}_{2}$ sample have been performed employing an array of 32 YAP detectors covering the angular
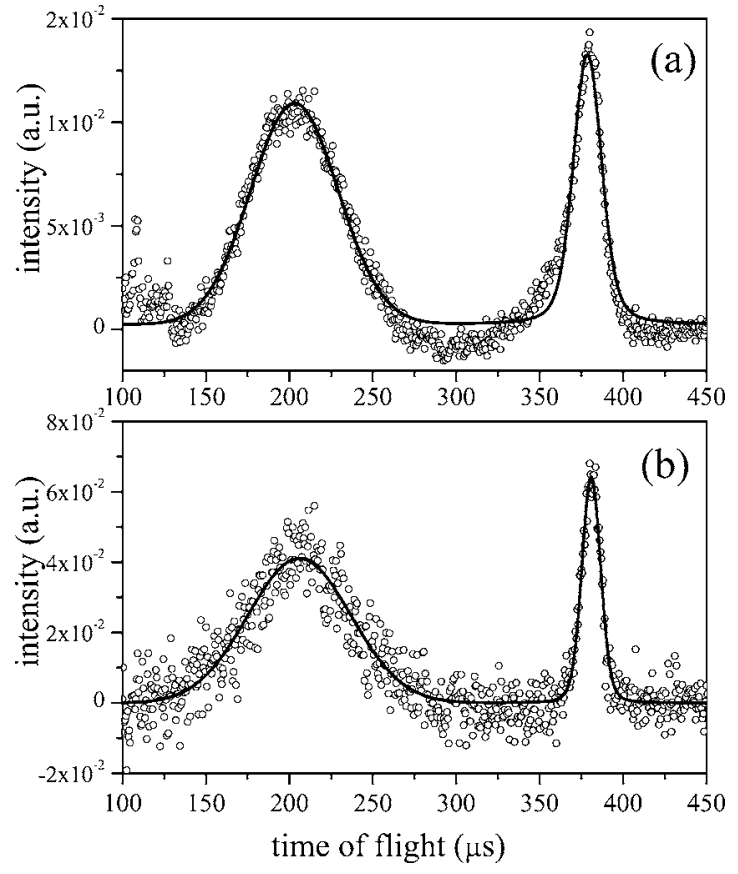

FIG. 8. Experimental TOF spectrum from a $\mathrm{ZrH}_{2}$ sample in the time region of 100-450 $\mu$ s, acquired using (a) the standard RD configuration and (b) the FCT. The continuous line is a multipeak fit of the ${ }^{1} \mathrm{H}$ peak (low TOF) and ${ }^{91} \mathrm{Zr}+{ }^{27} \mathrm{Al}$ peak (high TOF).

range $45^{\circ} \leqslant q \vartheta \leqslant 70^{\circ}$. Each scintillator had an area of $20 \mathrm{~cm}^{2}$ and a thickness of $0.6 \mathrm{~cm}$. As in the previous measurements, both the analyzer and cycling foils were made of $12.5 \mu \mathrm{m}$ thick ${ }^{197} \mathrm{Au}$ foils.

The sample was a $\mathrm{ZrH}_{2}$ powder with a thickness of $1 \mathrm{~mm}$, enclosed in an aluminum sample container. The cycling foils employed for the FCT were cycled in and out of the scattered neutron beam using the movable aluminum frame of VESUVIO which surrounds the sample tank. The average distance between analyzer and cycling foils was approximately $0.4 \mathrm{~m}$, with $L_{1} \simeq 0.75 \mathrm{~m}$.

The foil-out DINS spectrum, equivalent to a standard RD spectrum, acquired with the YAP detector placed at $2 \vartheta$ $\simeq 65^{\circ}$ is shown in Fig. 8(a). The peaks located at about 380 and $202 \mu$ s are, respectively, the ${ }^{91} \mathrm{Zr}+{ }^{27} \mathrm{Al}$ and ${ }^{1} \mathrm{H}$ recoil signals corresponding to a final neutron energy of $4.906 \mathrm{eV}$. A background correction has been applied by fitting the TOF spectrum in the 50-500 $\mu$ s region, excluding the peak regions and subtracting the fitted function from the data. In this case the background fit was not reliable in the region between the ${ }^{91} \mathrm{Zr}+{ }^{27} \mathrm{Al}$ and ${ }^{1} \mathrm{H}$ peaks $(250-350 \mu \mathrm{s})$. The estimated width of the ${ }^{91} \mathrm{Zr}+{ }^{27} \mathrm{Al}$ peak is about $20 \mu \mathrm{s}$ FWHM, while the width of the ${ }^{1} \mathrm{H}$ peak is about $72 \mu$ s FWHM. Figure 8(b) shows the DINS spectrum acquired with the same YAP detector configuration as the measurements shown in Fig. 8(a), but now employing the FCT. In this case the FWHM of the ${ }^{91} \mathrm{Zr}+{ }^{27} \mathrm{Al}$ peak is about $13 \mu \mathrm{s}$, while the width of the ${ }^{1} \mathrm{H}$ peak is circa $64 \mu$ s FWHM. The inherent background subtraction has again been very effective, even in the critical region between the peaks. Furthermore the change in the line shape of the recoil peaks, due to a sensible reduction of the intensity of the tails, provides an appreciable separation of the two scattering signals. 
TABLE I. Summary of the widths of the recoil peaks $\left(\mathrm{FWHM}_{P}\right)$ for the various samples used in the test experiments of the foil cycling technique (FCT). The first column lists the samples and the second column their individual components. The third column shows the widths provided by standard resonance detector (RD) measurements, while the fourth column lists the widths obtained utilizing the FCT.

\begin{tabular}{lccc}
\hline \hline Sample & Recoiling mass & $\begin{array}{c}\mathrm{FWHM}_{P}^{(\mathrm{RD})} \\
(\mu \mathrm{s})\end{array}$ & $\begin{array}{c}\mathrm{FWHM}_{P}^{(\mathrm{FCT})} \\
(\mu \mathrm{s})\end{array}$ \\
\hline Lead & ${ }^{207} \mathrm{~Pb}$ & 14 & 8 \\
Deuterium chloride & ${ }^{2} \mathrm{D}$ & 52 & 47 \\
& ${ }^{35} \mathrm{Cl}+{ }^{27} \mathrm{Al}$ & 18 & 10 \\
Zirconium hydride & ${ }^{1} \mathrm{H}$ & 72 & 64 \\
& ${ }^{91} \mathrm{Zr}+{ }^{27} \mathrm{Al}$ & 20 & 13 \\
\hline \hline
\end{tabular}

\section{SUMMARY}

A summary of the results obtained for the experiments on the $\mathrm{Pb}, \mathrm{DCl}$, and $\mathrm{ZrH}_{2}$ samples is given in Table I. In this table the FWHMs of the recoil peaks in time of flight are listed for the three different samples that have been discussed.

For large values of the scattering mass, the narrowing of the time distribution is more evident $(35 \%-40 \%)$, as the response function of the instrument is dominated by the energy component due to the analyzer's transfer function. For light elements (hydrogen, deuterium) the overwhelming contribution to the instrumental response comes from the intrinsic momentum distribution, so that the effect of the improvement of the resolution function does not exceed $10 \%-12 \%$ in the cases examined.

An important feature of the FCT is a very effective subtraction of the background in the whole time region of interest for DINS experiments, as successfully demonstrated by all measurements.

Apart from narrowing the FWHM of the recoil peaks, the FCT also gives an appreciable reduction of the tails of the recoil peaks. This is very important in order to separate the scattering signals from different masses, especially when hydrogen containing samples are investigated in terms of momentum distribution line shape or in terms of variation of the scattering intensity at high $\hbar \omega$ and $q .{ }^{33-35}$

In this article the case of analyzer and cycling foils of equal thickness is discussed, as this choice is a good compromise between resolution improvement and loss of counting efficiency. Other choices are conceivable though. For example, in the cases where the resolution is not so critical, so that FCT will be mainly used for background subtraction, the counting efficiency can be improved by using a thicker cycling foil. Vice versa, if energy resolution is of paramount importance, a thinner cycling foil can be used at the cost of counting efficiency. Alternatively, the cycling foil could be cooled. If the cycling foil were cooled, the background and wings of the peaks would subtract as with room temperature foils, but the overall width of the peak would be narrower and the net intensity near the peak would be even greater than with room temperature foils.

\section{DISCUSSION}

A novel technique has been introduced for improving the resolution function of a neutron spectrometer like VESUVIO operating in the resonance detector configuration. This technique, referred to as the foil cycling technique (FCT), utilizes an additional foil properly cycled in and out of the scattered neutron beam. Besides improving the resolution function, FCT also provides an effective background subtraction method. Both the improvement of the resolution function and the effective background subtraction method are considerable advantages for fine line shape analysis on the momentum distribution of the condensed matter systems probed by deep inelastic neutron scattering (DINS).

DINS experiments have been performed at the VESUVIO spectrometer on a variety of samples (lead, deuterium chloride, and zirconium hydride) to test the reliability and effectiveness of the FCT. These experiments have confirmed the appreciable improvement in the resolution function of the spectrometer. In the future, a detailed analysis of the line shape of the effective transfer function will be performed in order to make FCT an important tool for the analysis of DINS measurements. Furthermore, investigations into the application of FCT for high energy inelastic neutron scattering (HINS) experiments will be conducted.

The encouraging results obtained in these preliminary tests on FCT led to the design of a novel detector array based on YAP scintillators, envisaged for intermediate scattering angles $\left(25^{\circ} \leqslant 2 \vartheta \leqslant 70^{\circ}\right)$. This array will replace the neutron detection system used up to date for VESUVIO, based on ${ }^{6} \mathrm{Li}$-glass neutron counters in the RF configuration.

\section{ACKNOWLEDGMENTS}

This work was supported within the CNR-CCLRC Agreement No. 01/9001 concerning collaboration in scientific research at the spallation neutron source ISIS. The financial support of the Consiglio Nazionale delle Ricerche in this research is hereby acknowledged. The authors also acknowledge Professor C. A. Chatzidimitriou-Dreismann and Dr. T. Abdul-Redah for providing the DCl data for this analysis.

\footnotetext{
${ }^{1}$ R. Senesi et al., Physica B 276-278, S189 (2000).

${ }^{2}$ A. C. Evans, J. Mayers, D. N. Timms, and M. J. Cooper, Z. Naturforsch., A: Phys. Sci. 48, 425 (1993).

${ }^{3}$ C. Andreani, A. Pietropaolo, R. Senesi, G. Gorini, M. Tardocchi, N. Rhodes, E. M. Schooneveld, and A. D' Angelo, ISIS2003 Science Highlights.

${ }^{4}$ S. W. Lovesey, Theory of Neutron Scattering from Condensed Matter, 3rd ed. (Oxford University Press, Oxford, 1987); R. O. Simmons, Z. Naturforsch., A: Phys. Sci. 48, 415 (1993); G. K. Ivanov and Y. S. Sayasov, Sov. Phys. Usp. 9, 670 (1967); P. C. Hoemberg and P. M. Platzman, Phys. Rev. 152, 198 (1966).

${ }^{5}$ C. Andreani, D. Colognesi, J. Mayers, G. F. Reiter, and R. Senesi, Adv. Phys. 54, 377 (2005).

${ }^{6}$ A. C. Evans, J. Mayers, D. N. Timms, and M. J. Cooper, Z. Naturforsch., A: Phys. Sci. 48, 425 (1993); R. J. Newport, M. P. Paoli, V. T. Pugh, R. N. Sinclair, A. D. Taylor and W. G. Williams, Proceedings of the Eighth Meeting of the International Collaboration on Advanced Neutron Sources (ICANS VIII), Rutherford Appleton Laboratory Report No. RAL-85-110, 1985, p. 562.

${ }^{7}$ A. L. Fielding and J. Mayers, Nucl. Instrum. Methods Phys. Res. A 480, 680 (2002).

${ }^{8}$ D. R. Allen, E. W. J. Mitchell, and R. N. Sinclair, J. Phys. E 13, 639
} 
(1983); L. Cser, N. Kroó, P. Pacher, V. G. Simkin, and E. V. Vasilieva, Nucl. Instrum. Methods 179, 515 (1981).

${ }^{9}$ H. Rauh and N. Watanabe, Nucl. Instrum. Methods Phys. Res. A 228, 147 (1984); N. Watanabe, Proceedings of the 1984 Workshop on High Energy Excitations in Condensed Matter, 13-15 February 1984, Los Alamos National Laboratory Report No. LA-10227-C, Vol 1; J. M. Carpenter, N. Watanabe, S. Ikeda, Y. Masuda, S. Sato, and H. Rauh, Physica B \& C 120, 126 (1983).

${ }^{10}$ P. A. Seeger, A. D. Taylor, and R. M. Brugger, Nucl. Instrum. Methods Phys. Res. A 240, 98 (1985).

${ }^{11}$ C. Andreani, D. Colognesi, E. Degiorgi, A. Filabozzi, M. Nardone, E. Pace, A. Pietropaolo, and R. Senesi, Nucl. Instrum. Methods Phys. Res. A 497, 535 (2003).

${ }^{12}$ R. N. Sinclair, M. C. Moxon, and J. M. Carpenter, Bull. Am. Phys. Soc. 22, 101 (1977).

${ }^{13}$ R. K. Crawford, Proceedings of the IX Meeting of the International Collaboration on Advanced Neutron Sources (ICANS IX), 1986 (unpublished), Vol. 1, p. 397.

${ }^{14}$ C. Andreani, A. Pietropaolo, R. Senesi, G. Gorini, M. Tardocchi, A. Bracco, N. Rhodes, and E. Schooneveld, Nucl. Instrum. Methods Phys. Res. A 481, 509 (2002).

${ }^{15}$ C. Andreani et al., Appl. Phys. A: Mater. Sci. Process. 78, 903 (2003).

${ }^{16}$ A. Pietropaolo et al., Proceedings of the 11th International Symposium on Capture Gamma-Ray Spectroscopy and Related Topics (World Scientific, Singapore, 2003), p. 555.

${ }^{17}$ M. Tardocchi et al., Nucl. Instrum. Methods Phys. Res. A 526, 477 (2004).

${ }^{18}$ M. Tardocchi, G. Gorini, A. Pietropaolo, C. Andreani, R. Senesi, N. Rhodes, and E. M. Schooneveld, Rev. Sci. Instrum. 75, 4880 (2004).

${ }^{19}$ A. Pietropaolo, C. Andreani, G. Gorini, J. Mayers, E. Perelli-Cippo, N. J. Rhodes, E. M. Schooneveld, R. Senesi, M. Tardocchi, JINST 1, 04001 (2006).

${ }^{20}$ C. Andreani, G. Gorini, E. Perelli-Cippo, A. Pietropaolo, N. J. Rhodes, E.
M. Schooneveld, R. Senesi, and M. Tardocchi, Appl. Phys. Lett. 85, 5454 (2004).

${ }^{21}$ A. Pietropaolo, R. Senesi, M. Tardocchi, C. Andreani, and G. Gorini, Physica B 350, e857 (2004).

${ }^{22}$ E. Perelli-Cippo et al., Physica B (to be published).

${ }^{23}$ E. Perelli-Cippo, G. Gorini, M. Tardocchi, C. Andreani, A. Pietropaolo, R. Senesi, N. J. Rhodes, and E. M. Schoonveld, Appl. Phys. A: Mater. Sci. Process. (to be published).

${ }^{24}$ R. Senesi, Proceedings of the 17 th Meeting of the International Collaboration on Advanced Neutron Sources, Santa Fe, NM, 25-29 April 2005 (unpublished).

${ }^{25}$ A. Pietropaolo, C. Andreani, D. Fernandez-Cañoto, V. Garbuio, G. Gorini, S. Imberti, E. Perelli-Cippo, R. Senesi, and M. Tardocchi, Proceedings of the 17th Meeting of the International Collaboration on Advanced Neutron Sources (ICANS XVII), Santa Fe, NM, 25-29 April 2005, Vol. III, p. 898.

${ }^{26}$ A. Pietropaolo et al., internal report (unpublished).

${ }^{27}$ C. G. Windsor, Pulsed Neutron Scattering (Taylor \& Francis, London, 1981).

${ }^{28}$ G. Breit and E. Wigner, Phys. Rev. 49, 519 (1936).

${ }^{29}$ W. E. Lamb, Jr., Phys. Rev. 55, 190 (1939).

${ }^{30}$ M. S. Nelkin and D. E. Parks, Phys. Rev. 119, 1060 (1960).

${ }^{31}$ C. Andreani, G. Baciocco, R. S. Holt, and J. Mayers, Nucl. Instrum. Methods Phys. Res. A 276, '297 (1989).

${ }^{32}$ S. Imberti, C. Andreani, V. Garbuio, G. Gorini, A. Pietropaolo, R. Senesi, and M. Tardocchi, Nucl. Instrum. Methods Phys. Res. A 552, 463 (2005).

${ }^{33}$ C. A. Chatzidimitriou-Dreismann, T. A. Redah, R. M. F. Streffer, and J. Mayers, Phys. Rev. Lett. 79, 2839 (1997).

${ }^{34}$ E. B. Karlsson, C. A. Chatzidimitriou-Dreismann, T. Abdul-Redah, R. M. F. Streffer, B. Hjörvarsson, J. Öhrmalm, and J. Mayers, Europhys. Lett. 46, 617 (1999).

${ }^{35}$ R. Senesi, D. Colognesi, A. Pietropaolo, and T. Abdul-Redah, Phys. Rev. B 72, 054119 (2005). 